\title{
Multiple Positive Solutions of Second-Order Nonlinear Difference Systems with Repulsive Singularities
}

\author{
Shengjun Li $\mathbb{D}^{1}$ and Fang Zhang $\mathbb{D}^{2}$ \\ ${ }^{1}$ School of Science, Hainan University, Haikou 570228, China \\ ${ }^{2}$ Department of Mathematics, Changzhou University, Changzhou 213164, China \\ Correspondence should be addressed to Shengjun Li; shjli626@126.com
}

Received 31 March 2021; Revised 28 May 2021; Accepted 6 July 2021; Published 13 July 2021

Academic Editor: Liliana Guran

Copyright (C) 2021 Shengjun Li and Fang Zhang. This is an open access article distributed under the Creative Commons Attribution License, which permits unrestricted use, distribution, and reproduction in any medium, provided the original work is properly cited.

\begin{abstract}
We study the existence of positive solutions for second-order nonlinear repulsive singular difference systems with periodic boundary conditions. Our nonlinearity may be singular in its dependent variable. The proof of the main result relies on a fixed point theorem in cones and a nonlinear alternative principle of Leray-Schauder; the result is applicable to the case of a weak singularity as well as the case of a strong singularity. An example is given; some recent results in the literature are improved and generalized.
\end{abstract}

\section{Introduction}

Difference systems are widely used in modeling real-life phenomena [1] and references therein. In this paper, we establish the existence positive solutions for the following nonlinear difference systems:

$$
-\Delta[p(n-1) \Delta x(n-1)]+q(n) x(n)=f(n, x(n))+e(n),
$$

with the boundary conditions:

$$
x(0)=x(T), p(0) \Delta x(0)=p(T) \Delta x(T)
$$

where $q(n)=\operatorname{diag}\left(q_{1}(n), q_{2}(n), \cdots, q N(n)\right), p(n)=\operatorname{diag}\left(p_{1}(\right.$ $\left.n), p_{2}(n), \cdots, p N(n)\right), e=\left(e_{1}, e_{2}, \cdots, e_{N}\right) T$, and $f=\left(f_{1}, f_{2}, \cdots\right.$ , $f N) T, N \geq 1$. By a periodic solution, we mean a function $x$ $=\left(x_{1}, x_{2}, \cdots, x_{N}\right) T$, solving (1) and (2) and such that $x(n)$ $\neq 0$ for all $n$. We call boundary condition (2) the periodic boundary conditions which are important representatives of nonseparated boundary conditions. For convenience, we denote by $\mathbb{Z}, \mathbb{N}$, and $\mathbb{R}$ the sets of all integer numbers, natural numbers, and real numbers, respectively. For $a, b \in \mathbb{Z}$, let $\mathbb{Z}$ $(a)=\{a, a+1, \cdots\}, \mathbb{Z}[a, b]=\{a, a+1, \cdots, b\}$ when $a \leq b$. As usual, $\Delta$ denotes the forward difference operator defined by

$$
\Delta x(n)=x(n+1)-x(n) .
$$

In particular, the nonlinearity $f(x, x(n)): \mathbb{N} \times \mathbb{R}^{N} \backslash\{0\}$ $\longrightarrow \mathbb{R}^{N}$ may have a repulsive singularity at $x=0$, from the physical explanation, which means that $\lim _{x \rightarrow 0} f_{i}(n, x)=+\infty$, uniformly in $n \in \mathbb{Z}[1, T], i=1,2, \cdots, N$.

Such repulsive singularity appears in many problems of applications such as the Brillouin focusing systems and nonlinear elasticity [2].

System (1) can be viewed as a discretization of the following more general class of the Sturm singular second-order differential system:

$$
-(p(t) y 0) 0+q(t) y=f(t, y)+e(t) .
$$

Such systems, even in case $p \equiv 1$, where they are referred to as being of Klein-Gordon or Schrödinger type, appear in many scientific areas including fluid mechanics, gas dynamics, and quantum field theory. During the last few decades, the study of the existence of periodic solutions for singular differential equations has deserved the attention of many researchers [3-11]. Tracing back to 1987, Lazer and Solimini 
[5] investigated the singular model:

$$
x^{\prime \prime}+\frac{h(t)}{x^{\lambda}}=g(t)
$$

where $\lambda>0, h, g$ are $T$-periodic functions and the mean value of $g$ is negative, $\bar{g}<0$. One of the common conditions to guarantee the existence of positive periodic solution is a so-called strong force condition (corresponds to the case $\lambda$ $\geq 1$ in $(5))[11,12]$. For example, if we consider the system:

$$
\ddot{x}+\nabla V_{x}(t, x)=f(t),
$$

with $V(t, x)=1 /|x|^{a}$; the strong force condition holds for $\alpha$ $\geq 2$. On the other hand, the existence of positive periodic solutions of the singular differential equations has been established with a weak force condition (corresponds to the case $0<\lambda<1$ in (5)) [13-15].

From then on, some classical tools have been used to study singular differential equations in the literature, including the degree theory $[6,11,16]$, the method of the upper and lower solutions [8, 17], Schauder's fixed point theorem [14], some fixed point theorems in cones for completely continuous operators $[13,18]$, and a nonlinear Leray-Schauder alternative principle [19].

For the existence of periodic solutions of difference equations, some results have been obtained using the variational methods or the topological methods [1, 20-25]. For example, by minimax principle, Guo and Yu [23] discussed the existence of periodic solutions for difference equation:

$$
-\Delta^{2} x(n-1)+f(n, x(n))=0
$$

where the nonlinearity $f$ is of superlinear or sublinear growth at infinity. Based on the method of the upper and lower solutions, Atici and Cabada [21] studied the existence of periodic solutions for difference equation:

$$
-\Delta^{2} x(n-1)+q(n) x(n)=f(n, x(n))
$$

In [26], Zhou and Liu investigated the following autonomous difference equations:

$$
\Delta^{2} x(n-1)+f(x(n))=0
$$

By Conley index theory, the author showed that the suitable assumptions of asymptotically linear nonlinear are enough to guarantee the existence of periodic solutions.

In this paper, we establish two different existence results of positive periodic solutions for (1) and (2) and proof of the existence of positive solutions; the first one is based on an application of a nonlinear alternative of Leray-Schauder, which has been used by many authors [19, 27, 28] and references therein; the second one is based on a fixed point theorem in cones. Our main motivation is to obtain new existence results for positive periodic solutions of the system:

$$
\left\{\begin{array}{l}
-\Delta\left[p_{1}(n-1) \Delta x(n-1)\right]+q_{1}(n) x(n)=\left(x^{2}+y^{2}\right)^{-a / 2}+\mu\left(x^{2}+y^{2}\right)^{\beta / 2}+e_{1}(n) \\
-\Delta\left[p_{2}(n-1) \Delta y(n-1)\right]+q_{2}(n) y(n)=\left(x^{2}+y^{2}\right)^{-a / 2}+\mu\left(x^{2}+y^{2}\right)^{\beta / 2}+e_{2}(n)
\end{array}\right.
$$

Here, we emphasize that the new results are applicable to the case of a strong singularity as well as the case of a weak singularity and that $e$ does not need to be positive.

The rest of this paper is organized as follows. In Section 2, some preliminary results will be given. In Section 3, we will state and prove the main results. We will use the notation $\mathbb{R}_{+}^{N}=\left\{x \in \mathbb{R}^{N}: x_{i} \geq 0\right.$ for each $\left.i=1,2, \cdots, N\right\}$, for $x=\left(x_{1}\right.$, $\left.\cdots, x_{N}\right), y=\left(y_{1}, \cdots, y_{N}\right)$, we write $x \geq y$, if $x-y=\left(x_{1}-y_{1}, \cdots\right.$ , $\left.\mathrm{x}_{N}-Y_{N}\right) \in \mathbb{R}_{+}^{N}$. We say that a function $\varphi: \mathbb{R}^{N} \longrightarrow \mathbb{R}$ is nondecreasing if $\varphi(x) \geq \varphi(y)$ for $x, y \in \mathbb{R}^{N}$ with $x \geq y$. For a given function $p$ defined on $\mathbb{Z}[0, T]$, we denote its maximum and minimum by $p^{*}$ and $p_{*}$, respectively.

\section{Preliminaries}

For $i=1,2, \cdots, N$, let us denote by $\varphi_{i}(n)$ and $\psi_{i}(n)$ the solutions of the corresponding homogeneous equations:

$$
-\Delta\left[p_{i}(n-1) \Delta x(n-1)\right]+q_{i}(n) x(n)=0, n \in \mathbb{Z}[1, T],
$$

satisfying the initial conditions:

$$
\varphi_{i}(0)=\varphi_{i}(1)=0 ; \psi_{i}(0)=0, p_{i}(0) \psi_{i}(1)=1 .
$$

Let

$$
D_{i}=\varphi_{i}(T)+p_{i}(T) \Delta \psi_{i}(T)-2
$$

Throughout this paper, we always assume that

$(\mathrm{H})$ For each $i=1,2, \cdots, N, p_{i}(n)>0, q_{i}(n) \geq 0, q_{i}(\cdot) 6 \not \equiv 0$ , $n \in Z[1, T]$

Lemma 1 (see [29]). If $(H)$ holds, then $D_{i}>0$. 
Lemma 2 (see [29]). Assume (H) holds. For the solution of the the formula problem:

$$
\left\{\begin{array}{l}
-\Delta\left[p_{i}(n-1) \Delta x(n-1)\right]+q_{i}(n) x(n)=e_{i}(n), n \in \mathbb{Z}[1, T], \\
x(0)=x(T), p_{i}(0) \Delta x(0)=p_{i}(T) \Delta x(T),
\end{array} \quad x(n)=\sum_{s=1}^{T} G_{i}(n, s) e_{i}(s),\right.
$$

(14) holds, where

$G_{i}(n, s)=\frac{\psi_{i}(T)}{D_{i}} \varphi_{i}(n) \varphi_{i}(s)-\frac{p_{i}(T) \Delta \varphi_{i}(T)}{D_{i}} \psi_{i}(n) \psi_{i}(s)+ \begin{cases}\frac{p_{i}(T) \Delta \psi_{i}(T)-1}{D_{i}} \varphi_{i}(n) \psi_{i}(s)-\frac{\varphi_{i}(T)-1}{D_{i}} \varphi_{i}(s) \psi_{i}(n), & 0 \leq s \leq n \leq T+1, \\ \frac{p_{i}(T) \Delta \psi_{i}(T)-1}{D_{i}} \varphi_{i}(s) \psi_{i}(n)-\frac{\varphi_{i}(T)-1}{D_{i}} \varphi_{i}(n) \psi_{i}(s), & 0 \leq n \leq s \leq T+1,\end{cases}$

is the Green's function; the number $D_{i}$ is defined by (13).

Lemma 3 (see [29]). Under condition (H), the Green's function $G_{i}(n, s)$ of the boundary value problem (14) is positive, i.e., $G_{i}(n, s)>0$ for $n, s \in Z[0, T]$.

We denote

$$
A_{i}=\min _{n, s \in Z[0, T]} G_{i}(n, s), B_{i}=\max _{n, s \in Z[0, T]} G_{i}(n, s), \sigma=A_{i} / B_{i} .
$$

Obviously, $B_{i}>A_{i}>0$ and $0<\sigma_{i}<1$.

Remark 4. If $p_{i}(t)=1, q_{i}(t)=\alpha>0$, then Green's function $G_{i}(n, s)$ of the boundary value problem (14) has the form:

$$
G_{i}(n, s)= \begin{cases}\frac{\beta^{n-s}+\beta^{s-n+N}}{\left(\beta-\beta^{-1}\right)\left(\beta^{n}-1\right)}, & 0 \leq s \leq n \leq T+1, \\ \frac{\beta^{s-n}+\beta^{n-s+N}}{\left(\beta-\beta^{-1}\right)\left(\beta^{n}-1\right)}, & 0 \leq n \leq s \leq T+1\end{cases}
$$

where $\beta=(\alpha+2+\sqrt{\alpha(\alpha+2)}) / 2$. If $n$ is even, a direct calculation shows that

$$
\begin{gathered}
A_{i}=\frac{2 \beta^{T / 2}}{\left(\beta-\beta^{-1}\right)\left(\beta^{T}-1\right)}, \\
B_{i}=\frac{1+\beta^{T}}{\left(\beta-\beta^{-1}\right)\left(\beta^{T}-1\right)}, \\
\sigma_{i}=\frac{2 \beta^{T / 2}}{1+\beta^{T}}<1 .
\end{gathered}
$$

\section{Main Results}

In this section, we state and prove the new existence results for (1). In order to prove our main results, the following nonlinear alternative of Leray-Schauder is needed, which can be found in [30].

Lemma 5. Assume $\Omega$ is a relatively compact subset of a convex set $E$ in a normed space $X$. Let $\mathscr{A}: \bar{\Omega} \longrightarrow E$ be a compact map with $0 \in \Omega$. Then, one of the following two conclusions holds:

(i) Thas at least one fixed point in $\Omega$

(ii) There exist $u \in \partial \Omega$ and $0<\lambda<1$ such that $u=\lambda \mathscr{A} u$

\section{Let}

$$
\begin{aligned}
X_{1} & =\{x: \mathbb{Z}[0, T+1] \longrightarrow \mathbb{R} \mid x(0)=x(T), p(0) \Delta x(0) \\
& =p(T) \Delta x(T)\} .
\end{aligned}
$$

Then, $X_{1}$ is a Banach space with the norm

$$
\|x\|=\max _{n \in Z[1, T]} x(n) .
$$

We take

$$
X=X_{1} \times X_{1} \times \cdots \times X_{1}(N \text { copies }),
$$

with the norm

$$
|x|=\max \left\{\left\|x_{1}\right\|,\left\|x_{2}\right\|, \cdots,\left\|x_{N}\right\|\right\} .
$$

Define

$$
\gamma_{i}(n)=\sum_{s=1}^{T} G_{i}(n, s) e_{i}(s),
$$

which corresponds to the unique solution of (14), and the 
operator $\mathscr{A}: X \longrightarrow X$ by $\mathscr{A}_{x}=\left(\mathscr{A}_{1} x, \mathscr{A}_{2} x, \cdots, \mathscr{A}_{N} x\right)^{T}$, where

$$
\left(\mathscr{A}_{i} x\right)(n)=\sum_{s=1}^{T} G_{i}(n, s) f_{i}(s, x(s)+\gamma(s)), i=1,2, \cdots, N .
$$

Now, we present the first existence result of the positive solution to problem (1).

Theorem 6. Suppose that condition $(H)$ holds and $\gamma_{*} \geq 0$. Furthermore, we assume that

$\left(H_{1}\right)$ For each constant $L>0$, there exists a function $\varphi L($ $n)>0$ for all $n \in \mathbb{Z}[1, T]$ such that each component $f_{i}$ of $f$ satisfies $f_{i}(n, x) \geq \varnothing_{L}(n)$ for all $(n,|x|) \in \mathbb{Z}[1, T] \times(0, L]$
$\left(H_{2}\right)$ For each component $f_{i}$ of $f$, there exist nonnegative functions $g_{i}(x), h_{i}(x)$, and $k_{i}(n)$ such that

$0 \leq f_{i}(n, x) \leq\left\{g_{i}(x)+h_{i}(x)\right\} k_{i}(n)$ for all $(n, x) \in \mathbb{Z}[1, T] \times \mathbb{R}_{+}^{N}\{0\}$,

and $g_{i}(x)>0$ is nonincreasing and $h_{i}(x) / g_{i}(x)$ is nondecreasing in $x$ and

$\left(\mathrm{H}_{3}\right)$ There exists a positive number $r$ such that $\sigma r+\gamma_{*}>0$

$$
\frac{r}{g_{i}\left(\gamma_{*}, \cdots, \gamma_{*}, \sigma_{i} r+\gamma_{*}, \gamma_{*}, \cdots, \gamma_{*}\right)\left\{1+\left(\left(h_{i}\left(r+\gamma^{*}, \cdots, r+\gamma^{*}\right)\right) /\left(g_{i}\left(r+\gamma^{*}, \cdots, r+\gamma^{*}\right)\right)\right)\right\}}>K_{i}^{*},
$$

for all $i=1,2, \cdots, N$. Here,

$$
\begin{gathered}
K_{i}(n)=\sum_{s=1}^{T} G_{i}(n, s) k_{i}(s), \\
\sigma=\min _{i=1,2, \cdots, N}\left\{\sigma_{i}\right\}, \\
\gamma^{*}=\min _{i, n} \gamma(n), \\
\gamma^{*}=\max _{i, n} \gamma(n) .
\end{gathered}
$$

Then, (1) and (2) has at least one positive periodic solution $x$ with $x(n)>\gamma(n)$ for all $n \in Z[0, T]$ and $0<|x-\gamma|<r$.

Proof. We first show that

$$
-\Delta[p(n-1) \Delta x(n-1)]+q(n) x(n)=f(n, x(n)+\gamma(n)),
$$

together with (2) has a positive solution $x$ satisfying $x(n)+$ $\gamma(n)$ for $n \in Z[0, T]$ and $0<|x|<r$. If this is true, it is easy to see that $u(n)=x(n)+\gamma(n)$ will be a positive solution of (1) and (2) with $0<|u-\gamma|<r$ since

$$
\begin{aligned}
& -\Delta[p(n-1) \Delta u(n-1)]+q(n) u(n)=-\Delta[p(n-1) \Delta(x(n-1) \\
& \quad+\gamma(n-1))]+q(n)(x(n)+\gamma(n))=f(n, x(n)+\gamma(n)) \\
& \quad+e(n)=f(n, u(n))+e(n) .
\end{aligned}
$$

Since $\left(\mathrm{H}_{3}\right)$ holds, let $J_{0}=\left\{j_{0}, j_{0}+1, \cdots\right\}$, we can choose $j_{0} \in\{1,2, \cdots\}$ such that $1 / J_{0} \leq \sigma r+\gamma_{*}$ and

$g_{i}\left(\gamma_{*}, \cdots, \gamma_{*}, \sigma_{i} r+\gamma_{*}, \gamma_{*}, \cdots, \gamma_{*}\right)\left\{1+\frac{h_{i}\left(r+\gamma^{*}, \cdots, r+\gamma^{*}\right)}{g_{i}\left(r+\gamma^{*}, \cdots, r+\gamma^{*}\right)}\right\} K_{i}^{*}+\frac{1}{j_{0}}<r$,

for all $i=1,2, \cdots, N$.

Fix $j \in J_{0}$. Consider the family of systems

$$
\begin{aligned}
-\Delta & {[p(n-1) \Delta x(n-1)]+q(n) x(n)=\lambda f^{j}(n, x(n)+\gamma(n)) } \\
& +\frac{q(n)}{j}, n \in \mathbb{Z}[1, N],
\end{aligned}
$$

where $\lambda \in[0,1]$ and for each $i=1,2, \cdots, N$,

$f_{i}^{j}(n, x)= \begin{cases}f_{i}(n, x), & \text { if } x \geq \frac{1}{j}, \\ f_{i}\left(n, x_{1}, \cdots, x_{i-1}, \frac{1}{j}, x_{i}+1, \cdots, x_{N}\right), & \text { if } x \leq \frac{1}{j} .\end{cases}$

Problem (29) and (2) are equivalent to the following fixed point problem:

$$
x_{i}(n)=\lambda \sum_{s=1}^{T} G_{i}(n, s) f_{i}^{j}(s, x(s)+\gamma(s))+\frac{1}{j}=\lambda\left(\mathscr{A}_{i}^{j} x\right)(n)+\frac{1}{j}
$$

for each $i=1,2, \cdots, N$, here, we used the fact

$$
\sum_{s=1}^{T} G_{i}(n, s) q_{i}(s) \equiv 1, i=1,2, \cdots, N \text {. }
$$


We claim that any fixed point $x$ of (34) for any $\lambda \in[0,1]$ must satisfy $|x| \neq r$. Otherwise, assume that $x$ is a fixed point of (34) for some $\lambda \in[0,1]$ such that $|x|=r$. Without loss of generality, we assume that $\left|x_{l}\right|=r$ for some $l=1,2, \cdots, N$.

Thus, we have

$$
\begin{gathered}
x_{l}(n)-\frac{1}{j}=\lambda \sum_{s=1}^{N} G_{l}(n, s) f_{l}^{j}(n, x(s)+\gamma(s)) d s \geq \lambda A_{l} \sum_{s=1}^{T} f_{l}^{j}(n, x(s) \\
+\gamma(s)) d s=\sigma_{l} B_{l} \lambda \sum_{s=1}^{T} f_{l}^{j}(n, x(s) \\
+\gamma(s)) d s \geq \sigma_{l} \max _{n}\left\{\lambda \sum_{s=1}^{T} G_{l}(n, s) f_{l}^{j}(n, x(s)+\gamma(s)) d s\right\} \\
=\sigma_{l}\left\|x_{l}-\frac{1}{j}\right\| .
\end{gathered}
$$

Hence, for all $n \in Z[1, T]$, we have

$$
x_{l}(n) \geq \sigma_{l}\left\|x_{l}-\frac{1}{j}\right\|+\frac{1}{j} \geq \sigma_{l}\left(\left\|x_{l}\right\|-\frac{1}{j}\right)+\frac{1}{j} \geq \sigma_{l} r .
$$

Therefore,

$$
x_{l}(n)+\gamma_{l}(n) \geq \sigma_{l} r+\gamma_{*}>\frac{1}{j} .
$$
, $T$,

Using (34), we have from condition $\left(\mathrm{H}_{2}\right)$, for all $n \in Z[1$

$$
\begin{aligned}
x_{l}(n)= & \lambda \sum_{s=1}^{T} G_{l}(n, s) f_{l}^{j}(s, x(s)+\gamma(s))+\frac{1}{j} \\
= & \lambda \sum_{s=1}^{T} G_{l}(n, s) f_{l}(s, x(s)+\gamma(s))+\frac{1}{j} \\
\leq & \sum_{s=1}^{T} G_{l}(n, s) f_{l}(s, x(s)+\gamma(s))+\frac{1}{j} \\
\leq & \sum_{s=1}^{T} G_{l}(n, s) k_{l}(s) g_{l}(x(s)+\gamma(s))\left\{1+\frac{h_{1}(x(s)+\gamma(s))}{g_{1}(x(s)+\gamma(s))}\right\} \\
\leq & g_{l}\left(\gamma_{*}, \cdots, \gamma_{*}, \sigma_{l} r+\gamma_{*}, \gamma_{*}, \cdots, \gamma_{*}\right) \\
& \cdot\left\{1+\frac{h_{l}\left(r+\gamma^{*}, \cdots, r+\gamma^{*}\right)}{g_{l}\left(r+\gamma^{*}, \cdots, r+\gamma^{*}\right)}\right\} K_{l}^{*}+\frac{1}{j_{0}} .
\end{aligned}
$$

Therefore,

$$
\begin{aligned}
r= & \left|x_{l}\right| \leq g_{l}\left(\gamma_{*}, \cdots, \gamma_{*}, \sigma_{l} r+\gamma_{*}, \gamma_{*}, \cdots, \gamma_{*}\right) \\
& \cdot\left\{1+\frac{h_{l}\left(r+\gamma^{*}, \cdots, r+\gamma^{*}\right)}{g_{l}\left(r+\gamma^{*}, \cdots, r+\gamma^{*}\right)}\right\} K_{l}^{*}+\frac{1}{j_{0}} .
\end{aligned}
$$

This is a contradiction to the choice of $j_{0}$, and the claim is proved.
From this claim, the nonlinear alternative of LeraySchauder guarantees that

$$
x(n)=\left(\mathscr{A}^{j} x\right)(n)+\frac{1}{j},
$$

has a fixed point, denoted by $x^{j}(n)$, in $B_{r}=\{x \in X:|x|<r\}$, i.e.,

$$
-\Delta[p(n-1) \Delta x(n-1)]+q(n) x(n)=f^{j}(n, x(n)+\gamma(n))+\frac{q(n)}{j},
$$

has a periodic solution $x^{j}$ with $\left|x^{j}\right|<r$.

Next, we claim that these solutions $x^{j}(n)+\gamma(n)$ have a uniform positive lower bound, that is, there exists a constant $\delta>0$, independent of $j \in J_{0}$, such that

$$
\min _{i, n}\left\{x_{i}^{j}(n)+\gamma_{i}(n)\right\} \geq \delta,
$$

for all $j \in J_{0}$. To see this, we know from $\left(\mathrm{H}_{1}\right)$ that there exists a continuous function $\phi_{r}+\gamma^{*}(n)$ such that each component $f_{i}$ of $f$ satisfies $f_{i}(n, x) \geq \phi_{r+\gamma^{*}}(n)$ for all $(n,|x|) \in \mathbb{Z}[1, T] \times$ $\left(0, r+\gamma^{*}\right]$. Now, let $x^{r+\gamma^{*}}(n)$ be the unique solution to

$$
-\Delta[p(n-1) \Delta x(n-1)]+q(n) x(n)=\Phi(n),
$$

with (2), here $\Phi(n)=\left(\phi_{r+\gamma^{*}}(n), \cdots, \phi_{r+\gamma^{*}}(n)\right)^{T}$. Then, we have

$$
x_{i}^{r+\gamma^{*}}(n)+\gamma_{i}(n)=\sum_{s=1}^{T} G_{i}(n, s) \phi_{r+\gamma^{*}}(s)+\gamma_{i}(n) \geq \Phi_{*}+\gamma_{*}>0
$$

for each $i=1, \cdots, N$, here

$$
\Phi_{*}=\inf _{n} \Phi_{i}(n), \Phi_{i}(n)=\sum_{s=1}^{T} G_{i}(n, s) \phi_{r+\gamma^{*}}(s) .
$$

Next, we show that (43) holds for $\delta=\Phi_{*}+\gamma_{*}>0$. To see this, for each $i=1, \cdots, N$, since $x_{i}^{j}(n)+\gamma_{i}(n) \leq r+\gamma^{*}$ and $x_{i}^{j}$ $(n)+\gamma_{*} \geq 1 / j$, we have

$$
\begin{aligned}
x_{i}^{j}(n)+\gamma_{i}(n)=\sum_{s=1}^{T} G_{i}(n, s) f_{i}^{j}\left(s, x^{j}(s)+\gamma(s)\right)+\gamma_{i}(n) \\
+\frac{1}{j} \geq \sum_{s=1}^{T} G_{i}(n, s) \varnothing_{r+\gamma^{*}}+\gamma_{i}(n) \geq \Phi_{*}+\gamma_{*}:=\delta .
\end{aligned}
$$

The fact $|x(n)|<r$ and (43) show that for each $i=1,2$, $\cdots, N,\left\{x_{i}^{j}\right\}_{j \in J_{0}}$ is a bounded family on $\mathbb{Z}[1, T]$. Moreover, we have

$$
x_{i}^{j}(0)=x_{i}^{j}(T), p_{i}(0) \Delta x_{i}^{j}(0)=p_{i}(T) \Delta x_{i}^{j}(T),
$$


which implies that

$$
x_{i}^{j}(T+1)=\frac{p_{i}(0)}{p_{i}(T)} \Delta x_{i}^{j}(0)+x_{i}^{j}(T), j \in J_{0} .
$$

Thus, the Arzela-Ascoli theorem guarantees that $\left\{x_{i}^{j}\right\}$ $j \in J_{0}$ has a subsequence, $\left\{x_{i}^{j k}\right\} j_{k} \in J_{0}, k \in N$ converging uniformly on $\mathbb{Z}[0, T+1]$ to a function $x_{i}$. Let $x=\left(x_{1}, \cdots\right.$, $\left.x_{N}\right), x(n)$ satisfies $\delta \leq x_{i}(n)+\gamma_{i}(n)<r+\gamma^{*}$ for all $n \in \mathbb{Z}[1$ $, T]$ and $i=1, \cdots, N$. Moreover, $x_{i}^{j k}$ satisfies the integral equation:

$$
x_{i}^{j k}(n)=\sum_{s=1}^{T} G_{i}(n, s) f_{i}\left(s, x^{j k}(s)+\gamma(s)\right)+\frac{1}{j k}, i=1, \cdots, N
$$

Letting $k \longrightarrow \infty$, we arrive at

$$
x_{i}(n)=\sum_{s=1}^{T} G_{i}(n, s) f_{i}(s, x(s)+\gamma(s)), i=1,2, \cdots, N
$$

here, we have used the fact that $f(n, x)$ is with respect to $(n, x)$ with $n \in \mathbb{Z}[1, T]$ and $x>0$ satisfying $\delta \leq|x| \leq r+\gamma^{*}$. Therefore, $x$ is a positive periodic solution of (1) and satisfies $0<|x| \leq r$

Corollary 7. Assume that $(H)$ holds, $\alpha>0, \beta \geq 0$. Then, for each $e_{1}, e_{2}$ with $\gamma_{*} \geq 0$, we have

(i) if $\beta<1$, then (10) has at least one positive periodic solution for each $\mu>0$

(ii) if $\beta \geq 1$, then (10) has at least one positive periodic solution for each $0<\mu<\mu_{1}$, where $\mu_{1}$ is some positive constant

Proof. We will apply Theorem 6. To this end, assumption $\left(\mathrm{H}_{1}\right)$ is fulfilled by $\varnothing_{L}=(\sqrt{2 L})^{-\alpha}$. If we take

$$
\begin{aligned}
& g_{1}(x, y)=g_{2}(x, y)=\left(x^{2}+y^{2}\right)^{-\alpha / 2}, \\
& h_{1}(x, y)=h_{2}(x, y)=\mu\left(x^{2}+y^{2}\right)^{\beta / 2},
\end{aligned}
$$

and $k_{1}(n)=k_{2}(n)=1$, then $\left(\mathrm{H}_{2}\right)$ is satisfied.

Let

$$
\omega_{1}(n)=\sum_{s=1}^{T} G_{1}(n, s), \omega_{2}(n)=\sum_{s=1}^{T} G_{2}(n, s) .
$$

Then, the existence condition $\left(\mathrm{H}_{3}\right)$ becomes

$$
\mu<\frac{r\left[\left(\sigma_{i} r+\gamma_{*}\right)^{2}+\gamma_{*}^{2}\right]^{2 / \alpha}-\omega_{i}^{*}}{2^{(\alpha+\beta) / 2}\left(r+\gamma^{*}\right)^{\alpha+\beta}}, i=1,2,
$$

for some $r>0$. So, (10) has at least one positive periodic solution for

$$
0<\mu<\mu_{1}:=\min _{i=1,2} \sup _{r>0} \frac{r\left[\left(\sigma_{i} r+\gamma_{*}\right)^{2}+\gamma_{*}^{2}\right]^{2 / \alpha}-\omega_{i}^{*}}{2^{(\alpha+\beta) / 2}\left(r+\gamma^{*}\right)^{\alpha+\beta}}, i=1,2 .
$$

Note that $\mu_{1}=\infty$ if $\beta<1$ and $\mu_{1}<\infty$ if $\beta \geq 1$. We have (i) and (ii).

In more general, we can obtain the following result.

Corollary 8. Assume that $(H)$ holds and there exist functions $a, \widehat{a}, b, \widehat{b}$ and $\alpha, \beta>0$ such that, for $i=1,2, \cdots, N$,

$$
\frac{\alpha(n)}{|x|^{\alpha}}+b(n)|x|^{\beta} \leq f_{i}(n, x) \leq \frac{\widehat{\alpha}(n)}{|x|^{\alpha}}+\mu \widehat{b}(n)|x|^{\beta} .
$$

Then, for each e with $\gamma_{*} \geq 0$, we have

(i) if $\beta<1$, then (10) has at least one positive periodic solution for each $\mu>0$

(ii) if $\beta \geq 1$, then (10) has at least one positive periodic solution for each $0<\mu<\mu_{2}$, where $\mu_{2}$ is some positive constant

By using a fixed point theorem for compact maps on conical shells [31], we established the second positive periodic solution for (1). Recall that a compact operator means an operator which transforms every bounded set into a relatively compact set and introducing the definition of a cone.

Definition 9. Let $X$ be a Banach space and let $K$ be a closed, nonempty subset of $X$. $K$ is a cone if

(i) $\alpha u+\beta v \in K$ for all $u, v \in K$ and all $\alpha, \beta>0$

(ii) $u,-u \in K$ implies $u=0$

Lemma 10 (see [31]). Let $X$ be a Banach space and $K$ a cone in $X$. Assume $\Omega_{1}, \Omega_{2}$ are open subsets of $X$ with $0 \in \Omega_{1}, \bar{\Omega}_{1}$ $\in \Omega_{2}$. Let

$$
\Phi: K \cap\left(\bar{\Omega}_{2} \backslash \Omega_{1}\right) \longrightarrow K
$$

be a continuous and completely continuous operator such that

(i)

$$
\|\Phi x\| \leq\|x\| \text { for } x \in K \cap \partial \Omega_{1}
$$

(ii) There exist $\psi \in K \backslash\{0\}$ such that $x \neq \Phi x+\lambda \psi$ for $x$ $\in K \cap \partial \Omega_{2}$ and $\lambda>0$ 
Then, $F$ has a fixed point in $K \cap\left(\bar{\Omega}_{2} \backslash \Omega_{1}\right)$. The same conclusion remains valid if ( $i$ ) holds on $K \cap \partial \Omega_{2}$, and (ii) holds on $K \cap \partial \Omega_{1}$.

Define

$$
\begin{aligned}
K & =\left\{x=\left(x_{1}, \cdots, x_{N}\right) \in X: \min _{0 \leq n \leq T} x_{i}(n)\right. \\
& \left.\left.\geq \sigma_{i}\left\|x_{i}\right\|\right\} \text { for all } n \in Z[0, T], i=1, \cdots, N\right\} .
\end{aligned}
$$

Then, one can readily verify that $K$ is a cone in $X$.
Theorem 11. Suppose conditions $(H),\left(H_{1}\right)-\left(H_{3}\right)$ hold. Furthermore, assume that the following two conditions are satisfied:

$\left(\mathrm{H}_{4}\right)$ There exist continuous, nonnegative functions $g^{1}(x)$, $h^{1}(x)$ and $k^{1}(n)$ such that

$$
f_{i}(n, x) \geq\left\{g_{i}^{1}(x)+h_{i}^{1}(x)\right\} k_{i}^{1}(n) \text { for all }(n, x) \in[0, T] \times \mathbb{R}_{+}^{n}\{0\},
$$

where $g_{i}^{1}(x)>0$ is nonincreasing and $h_{i}^{1}(x) / g_{i}^{1}(x)$ is nondecreasing in $x$

$\left(\mathrm{H}_{5}\right)$ There exists $\mathrm{R}>r$ such that

$$
\frac{\sigma R}{g_{i}^{1}\left(R+\gamma^{*}, \cdots, R+\gamma^{*}\right)\left\{1+\left(\left(h_{i}^{1}\left(\sigma_{1} R+\gamma_{*}, \cdots, \sigma_{n} R+\gamma_{*}\right)\right) /\left(g_{i}^{1}\left(\sigma_{1} R+\gamma_{*}, \cdots, \sigma_{n} R+\gamma_{*}\right)\right)\right)\right\}} \leq K_{i *} .
$$

Then, problems (1) and (2) have another one positive periodic solution $\tilde{x}$ with $r<|\tilde{x}-\gamma| \leq R$.

Proof. Let $\mathscr{A} x=\left(\mathscr{A}_{1} x, \cdots, \mathscr{A}_{N} x\right)^{T}, \mathscr{A}_{i} x$ is given by (25), then, it is easy to verify that $\mathscr{A}$ is well defined and maps $X$ into $K$. Moreover, $\mathscr{A}$ is continuous and completely continuous, and let $K$ be a cone in $X$ defined by (59). Define the

$$
\Omega_{1}=\{x \in X:|x|<r\}, \Omega_{2}=\{x \in X:|x|<R\} .
$$

As in the proof of Theorem 3.1, we only need to show that (29) has a positive periodic solution $u \in X$ with $u(n)+$ $\gamma(n)>0$ and $r<|u| \leq R$. We claim that

(i)

$$
|\mathscr{A} x| \leq|x| \text { for } x \in K \cap \partial \Omega_{1}
$$

(ii) There exist $\psi \in K \backslash\{0\}$ such that $x \neq \mathscr{A} x+\lambda \psi$ for $x$ $\in K \cap \partial \Omega_{2}$ and $\lambda>0$

We start with (i). In fact, if $x \in K \cap \partial \Omega_{1}$, then $|x|=r$ and $\sigma_{i} r+\gamma_{*} \leq x_{i}(n)+\gamma(n) \leq r+\gamma^{*}$ for all $t \in[0, T]$. Fix $i \in\{1,2$, $\cdots, N\}$, thus, we have

$$
\begin{aligned}
\left(\mathscr{A}_{i} x\right)(t)= & \sum_{s=1}^{T} G_{i}(n, s) f_{i}(s, x(s)+\gamma(s)) \leq \sum_{s=1}^{T} G_{i}(n, s) k_{i}(s) g_{i}(x(s) \\
& +\gamma(s))\left\{1+\frac{h_{i}(x(s)+\gamma(s))}{g_{i}(x(s)+\gamma(s))}\right\} \leq g_{i}(x(s) \\
& +\gamma(s))\left\{1+\frac{h_{i}(x(s)+\gamma(s))}{g_{i}(x(s)+\gamma(s))}\right\} \sum_{s=1}^{T} G_{i}(n, s) k_{i}(s) \\
\leq & g_{i}\left(\gamma_{*}, \cdots, \gamma_{*}, \sigma_{i} r+\gamma_{*}, \gamma_{*}, \cdots, \gamma_{*}\right) \\
& \cdot\left\{1+\frac{h_{i}\left(r+\gamma^{*}, \cdots, r+\gamma^{*}\right)}{g_{i}\left(r+\gamma^{*}, \cdots, r+\gamma^{*}\right)}\right\} K_{i *}<r=|x| .
\end{aligned}
$$

Therefore, $\left\|\mathscr{A}_{i} x\right\| \leq|x|$ for each $i=1,2, \ldots, N$. This implies that (i) holds.

Next, we show that (ii) holds. Let $\psi \equiv(1, \cdots, 1)$, then $\psi \in K \backslash\{0\}$. Suppose that there exists $x \in K \cap \partial \Omega_{2}$ and $\lambda>0$ such that $x=\mathscr{A} x+\lambda \psi$. Since $x \in K \cap \partial \Omega_{2}$, then $\sigma_{i}$ $R+\gamma_{*} \leq x_{i}(n)+\gamma(n) \leq R+\gamma^{*}$ for all $n \in Z[0, T]$. As a result, it follows from $\left(\mathrm{H}_{4}\right)$ and $\left(\mathrm{H}_{5}\right)$ that, for all $n \in Z[$ $0, T]$,

$$
\begin{aligned}
x_{i}(n)= & \left(\mathscr{A}_{i} x\right)(n)+\lambda=\sum_{s=1}^{T} G_{i}(n, s) f_{i}(s, x(s)+\gamma(s)) d s+\lambda \\
\geq & \sum_{s=1}^{T} G_{i}(n, s) k_{i}^{l}(s) g_{1}^{l}(x(s)+\gamma(s))\left\{1+\frac{h_{i}^{l}(x(s)+\gamma(s))}{g_{i}^{l}(x(s)+\gamma(s))}\right\} \\
& +\lambda \geq g_{i}^{l}(x(s)+\gamma(s))\left\{1+\frac{h_{i}^{l}(x(s)+\gamma(s))}{g_{i}^{l}(x(s)+\gamma(s))}\right\} \sum_{s=1}^{T} G_{i}(n, s) k_{i}^{l}(s) \\
& +\lambda \geq g_{i}^{l}\left(R+\gamma^{*}, \cdots, R+\gamma^{*}\right)\left\{1+\frac{h_{i}^{l}\left(\sigma_{1} R+\gamma_{*}, \cdots, \sigma_{N} R+\gamma_{*}\right)}{g_{i}^{l}\left(\sigma_{1} R+\gamma_{*}, \cdots, \sigma_{N} R+\gamma_{*}\right)}\right\} K_{i *} \\
& +\lambda \geq \sigma R+\lambda .
\end{aligned}
$$

Hence, $\min _{0 \leq n \leq T} x_{i}(n)>\sigma R$; this is a contradiction and we prove the claim.

Now, Lemma 3.7 guarantees that $\mathscr{A}$ has at least one fixed point $x \in K \cap\left(\bar{\Omega}_{2} \backslash \Omega_{1}\right)$ with $r \leq|x| \leq R$.

Let us consider again the example (10) in Corollary 7 for the superlinear case.

Corollary 12. Assume in (10) that $p_{i}, q_{i}(i=1,2)$ satisfy $(H)$, for each $e_{1}, e_{2}$ with $\gamma_{*} \geq 0, \beta>1$. Then, for each $\mu$ with 0 $<\mu<\mu_{1}$, where $\mu_{1}$ is given as in Corollary 7 , problem (10) has at least two different positive solutions. To verify 
$\left(H_{4}\right)$, one may take

$$
\begin{gathered}
g_{1}^{1}(x, y)=g_{2}^{1}(x, y)=\frac{1}{2}\left(x^{2}+y^{2}\right)^{-\alpha / 2}, \\
h_{2}^{1}(x, y)=\frac{1}{2}\left(x^{2}+y^{2}\right)^{\beta / 2},
\end{gathered}
$$

and $k^{1}(n)_{1}=k_{2}^{1}(n)=1$. If $\beta>1$, then the existence condition $\left(\mathrm{H}_{5}\right)$ becomes

$$
\mu \geq \frac{2^{(\alpha+2) / 2}(R+\gamma *)^{\alpha} \sigma R-2 \omega_{i *}}{\left[\left(\sigma_{1} R+\gamma_{*}\right)^{2}+\left(\sigma_{2} R+\gamma_{*}\right)^{2}\right]^{(\alpha+\beta) / 2} \omega_{i *}}, i=1,2 .
$$

Since $\beta>1$, the right-hand side goes to 0 as $R \longrightarrow+$ $\infty$. Thus, for any given $0<\mu<\mu 1$, it is always possible to find such $R \gg r$ that (67) is satisfied. Thus, (10) has an additional positive periodic solution $\tilde{x}$.

Remark 13. We emphasize that our results are applicable to the case of a strong singularity as well as the case of a weak singularity since we only need $\alpha>0$. Moreover, $e$ does not need to be positive. In fact, using the assumption that the Green function is positive, one may readily verify that $\gamma_{*} \geq$ 0 is equivalent to the $\sum_{i=1}^{N} e_{i}(n) \geq 0, i=1,2, \cdots, N$.

Let us consider the 2 -dimensional system

$$
\left\{\begin{array}{l}
-\Delta^{2} x(n-1)+x(n)=\frac{\alpha(n)}{|x|^{\alpha}}+\mu b(n)|x|^{\beta}+e_{1}(n), \\
-\Delta^{2} y(n-1)+y(n)=\frac{\alpha(n)}{|x|^{\alpha}}+\mu b(n)|x|^{\beta}+e_{2}(n),
\end{array}\right.
$$

with

$$
e_{i}(n)=n\left(d_{i}-n\right), d_{i} \in \mathbb{R}, i=1,2 \text {. }
$$

Example 1. Assume that $\alpha>0, \beta>1, a(n)$, and $b(n)$ are positive functions, $e_{i}(n), i=1,2$ are given by (69) with

$$
d_{1}+2 d_{2} \geq 5
$$

Then, the results in Corollary 12 hold.

Proof. We only need show $\gamma_{*} \geq 0$, which is equivalent to

$$
\sum_{i=1}^{2} e_{i}(n) \geq 0
$$

Since $d_{1}+2 d_{2} \geq 5$, a direct computation show that

$$
\sum_{i=1}^{2} e_{i}(n) \geq 0 \sum_{i=1}^{2}=n\left(d_{i}-n\right) \geq 0 .
$$

\section{Conclusions}

In this paper, we study the periodic problem for nonlinear difference systems with a singularity of repulsive type in the case of $\gamma_{*} \geq 0$. The proofs of main results are based on a nonlinear alternative principle of Leray-Schauder and a fixed point theorem in cones. It is interesting that the singularity $f$ is applicable to the case of a weak singularity as well as the case of a strong singularity. In the next research, we will continue to study the periodic problem to the difference systems like (10) where $f$ may have attractive singularity at $x=0$ , and whether the condition $\gamma_{*} \geq 0$ can be removed.

\section{Data Availability}

The data used to support the findings of this study are included within the article.

\section{Conflicts of Interest}

The authors declare that they have no conflicts of interest.

\section{Acknowledgments}

This work is supported by the Hainan Provincial Natural Science Foundation of China (Grant No. 120RC450), National Natural Science Foundation of China (Grant No. 11861028), and Jiangsu Provincial Natural Science Foundation of China (Grant No. BK20201447).

\section{References}

[1] Z. Balanov, C. Garca-Azpeitia, and W. Krawcewicz, "On variational and topological methods in nonlinear difference equations," Communications on Pure \& Applied Analysis, vol. 17, no. 6, pp. 2813-2844, 2018.

[2] M. A. Delpino and R. F. Manasevich, "Infinitely many T-periodic solutions for a problem arising in nonlinear elasticity," Journal of Differential Equations, vol. 103, pp. 260-277, 1993.

[3] J. Alzabut and C. Tunc, "Existence of periodic solutions for Rayleigh equation with statedependent delay," Electronic Journal of Differential Equations, vol. 77, pp. 1-8, 2012.

[4] Z. Cheng and X. Cui, "Positive periodic solution to an indefinite singular equation," Applied Mathematics Letters, vol. 112, pp. 1-7, 2021.

[5] A. C. Lazer and S. Solimini, "On periodic solutions of nonlinear differential equations with singularities," Proceedings of the American Mathematical Society, vol. 99, no. 1, pp. 109-114, 1987.

[6] S. Li, F. Liao, and W. Xing, "Periodic solutions of Liénard differential equations with singularity," Electronic Journal of Differential Equations, vol. 151, pp. 1-12, 2015.

[7] S. Li, H. Luo, and X. Tang, "Periodic orbits for radially symmetric systems with singularities and semilinear growth," Results in Mathematics, vol. 72, no. 4, pp. 1991-2011, 2017.

[8] I. Rachůnková, M. Tvrdý, and I. Vrkoč, "Existence of nonnegative and nonpositive solutions for second order periodic boundary value problems," Journal of Differential Equations, vol. 176, pp. 445-469, 2001. 
[9] F. Wang and Y. Cui, "On the existence of solutions for singular boundary value problem of thirdorder differential equations," Mathematica Slovaca, vol. 60, no. 4, pp. 485-494, 2010.

[10] F. Wang, F. Zhang, and F. Wang, "The existence and multiplicity of positive solutions for second-order periodic boundary value problem," Journal of Function Spaces and Applications, vol. 2012, article 725646, pp. 1-13, 2012.

[11] P. Yan and M. Zhang, "Higher order nonresonance for differential equations with singularities," Mathematicsl Methods in the Applied Sciences, vol. 26, no. 12, pp. 1067-1074, 2003.

[12] Z. Cheng and J. Ren, "Periodic and subharmonic solutions for Duffing equation with a singularity," Discrete \& Continuous Dynamical Systems - A, vol. 32, no. 5, pp. 1557-1574, 2012.

[13] D. Franco and J. R. L. Webb, "Collisionless orbits of singular and nonsingular dynamical systems," Discrete \& Continuous Dynamical Systems - A, vol. 15, no. 3, pp. 747-757, 2006.

[14] D. Franco and P. J. Torres, "Periodic solutions of singular systems without the strong force condition," Proceedings of the American Mathematical Society, vol. 136, pp. 1229-1236, 2008.

[15] P. J. Torres, "Weak singularities may help periodic solutions to exist," Journal of Differential Equations, vol. 232, no. 1, pp. 277-284, 2007.

[16] M. Zhang, "A relationship between the periodic and the Dirichlet BVPs of singular differential equations," Proceedings of the Royal Society of Edinburgh, vol. 128, no. 5, pp. 10991114, 1998.

[17] D. Bonheure and C. De Coster, "Forced singular oscillators and the method of lower and upper solutions," Topological Methods in Nonlinear Analysis, vol. 22, no. 2, pp. 297-317, 2003.

[18] S. Li, F. Liao, and H. Zhu, "Multiplicity of positive solutions to second-order singular differential equations with a parameter," Boundary Value Problems, vol. 115, 2014.

[19] D. Jiang, J. Chu, and M. Zhang, "Multiplicity of positive periodic solutions to superlinear repulsive singular equations," Journal of Differential Equations, vol. 211, no. 2, pp. 282302, 2005.

[20] J. Alzabut, "Existence of periodic solutions for a type of linear difference equations with distributed delay," Advances in Difference Equations, vol. 2012, Article ID 53, 2012.

[21] F. M. Atici and A. Cabada, "Existence and uniqueness results for discrete second-order periodic boundary value problems," Computers \& Mathematcs with Applications, vol. 45, no. 6-9, pp. 1417-1427, 2003.

[22] P. Cheng and X. Tang, "Existence of homoclinic orbits for 2nth-order nonlinear difference equations containing both many advances and retardations," Journal of Mathematical Analysis and Applications, vol. 381, no. 2, pp. 485-505, 2011.

[23] Z. Guo and J. Yu, "The existence of periodic and subharmonic solutions of subquadratic second order difference equations," Journal of the London Mathematical Society, vol. 68, no. 2, pp. 419-430, 2003.

[24] S. Mobayen, "Adaptive global terminal sliding mode control scheme with improved dynamic surface for uncertain nonlinear systems," International Journal of Control, Automation and Systems, vol. 16, no. 4, pp. 1692-1700, 2018.

[25] X. Zhang and X. Tang, "Existence of nontrivial solutions for boundary value problems of second-order discrete systems," Mathematica Slovaca, vol. 61, no. 5, pp. 769-778, 2011.
[26] B. Zhou and C. Liu, "Applications of Conley index theory on difference equations with non-resonance," Applied Mathematics Letters, vol. 108, article 106500, 2020.

[27] J. Chu, P. J. Torres, and M. Zhang, "Periodic solutions of second order non-autonomous singular dynamical systems," Journal of Differential Equations, vol. 239, no. 1, pp. 196212, 2007.

[28] S. Li and Y. Wang, "Multiplicity of positive periodic solutions to second order singular dynamical systems," Mediterranean Journal of Mathematics, vol. 14, article 202, 2017.

[29] F. M. Atici and G. S. Guseinov, "Positive periodic solutions for nonlinear difference equations with periodic coefficients," Journal of Mathematical Analysis and Applications, vol. 232, no. 1, pp. 166-182, 1999.

[30] D. O'Regan, Existence Theory for Nonlinear Ordinary Differential Equations, Kluwer Academic, Dordrecht, 1997.

[31] D. Guo and V. Lakshmikantham, Nonlinear Problem in Abstract Cones, Academic Press, London, 1988. 